\title{
Asesoría de acercamiento a la práctica docente
}

\author{
Otoniel Viramontes Campos \\ Escuela Normal Rural Ricardo Flores Magón \\ leprechu@hotmail.com
}

\begin{abstract}
Resumen
Este trabajo de investigación trata sobre la caracterización de la función del asesor en el área: Acercamiento a la práctica en el programa educativo de la licenciatura de educación preescolar que se desarrolla en la escuela normal rural Ricardo Flores Magón de Saucillo, Chihuahua. El estudio se realiza a través de un enfoque cualitativo con el método etnográfico. Entre otras herramientas se utilizaron la encuesta, observación participante, entrevistas y análisis de diarios de trabajo de las estudiantes. En los resultados se percibieron dos vertientes en la caracterización de la función del asesor: la primera que es eminentemente académica que corresponde a el trabajo que se desarrolla en el aula y en las prácticas profesionales de las estudiantes; la segunda tiene que ver con el apoyo a las actividades extracurriculares que realizan las alumnas durante la formación profesional.
\end{abstract}

\section{Palabras clave}

Asesoramiento, práctica docente, formación de profesores.

\section{Introducción}

La práctica docente es un proceso complejo visto desde diferentes ángulos, este trabajo pretende hacerlo desde dentro de la propia práctica, y todavía más rico en el sentido de hacerlo en un espacio donde se forman los docentes.

En este caso se habla de la escuela Normal Rural Ricardo Flores Magón ubicada en Saucillo, Chihuahua, región caracterizada por ser eminentemente agrícola, con algunos negocios de diversa índole ya establecidos así como la instalación de maquilas que dan sustento y apoyo económico a la comunidad.

Los cursos de Escuela y contexto, Iniciación al trabajo escolar y los de Observación y práctica docente I, II, III y IV se incluyen dentro de la serie de espacios curriculares de acercamiento a la práctica docente. El mapa curricular define un acercamiento a los diferentes contextos que el propio estado cuenta, o simplemente aquellos a los que se pueda tener acceso, siempre y cuando éstos cuenten con características diversas, aquí la alumna observará cada uno de ellos y verá cómo es que se trabaja con este tipo de características.

En estos cursos es importante que los docentes de las escuelas normales tengan claridad sobre sus funciones de la asesoría en dichos cursos, toda vez que de su desempeño depende la parte central de la formación profesional de las estudiantes.

La observación y práctica se realiza en los jardines de niños de la educación básica. Como principio se establece que la práctica, 
que según Lella (2010), frecuentemente se concibe como la acción que se desarrolla en el aula y, dentro de ella, con especial referencia al proceso de enseñar. Pero también a la disposición de aprender y construir, por ende, a realizar una práctica institucional global y sus nexos insoslayables con el conjunto de la práctica social del docente. Es decir, descubren que el docente no sólo es eso, pero esto es tema de otra investigación.

La coordinación de las actividades de la observación y práctica docente de las alumnas requiere de directrices concretas que, aunque sí existen, no todas están explícitas en los planteamientos curriculares, porque además de lo que se establece en el programa educativo, existen una serie de actividades y funciones que se han percibido como necesarias para el desarrollo de la función del asesor de las actividades de acercamiento a la práctica docente de las estudiantes.

Estas competencias se desarrollan a través de la experiencia, de la observación de lo que hacen otros docentes de la escuela, de lo que plantea el programa educativo; pero también de las necesidades que se presentan inesperadamente para la organización, coordinación y asesoría de las actividades de acercamiento a la práctica docente.

A partir de la definición del perfil de egreso es conveniente establecer algunos criterios y orientaciones de orden académico que tienen dos propósitos: precisar los lineamientos más importantes que regulan los contenidos, la organización y la secuencia de las asignaturas $y$ otras actividades establecidas en el plan; y, en segundo lugar, definir ciertos rasgos comunes de las formas de trabajo académico y del desempeño del personal docente, que son indispensables para que los propósitos educativos se alcancen realmente en la práctica. Por esa razón se señala que un plan de estudios, por correcta que sea su formulación, sólo tiene la posibilidad de alcanzar sus objetivos cuando su aplicación se realiza en un ambiente educativo y bajo prácticas que son congruentes con las finalidades del plan, considerando para ello las adecuaciones necesarias para que las funciones del asesor de las asignaturas de acercamiento a la práctica, las explícitas y las implícitas, se atiendan en lo necesario de acuerdo a los requerimientos específicos y contextuales en que se desarrollan las actividades.

\section{Planteamiento}

Realizar una investigación aquí se puede dar en diferentes direcciones, pero en particular, y considerando los años de servicio y la propia novela escolar, se plantea la siguiente interrogante: ¿Cuáles son las actividades que realiza el maestro de observación y práctica docente (OPD)?.

\section{Preguntas de investigación.}

1. ¿Cuáles son las tareas que el maestro de OPD realiza? y ¿cuáles se sugieren en el programa educativo?

2. ¿Cómo enfrenta las situaciones que se le presentan?

3. ¿Cuáles son los problemas a los que se enfrenta el asesor de OPD?

4. ¿Cuáles son las actividades que tienen mayor incidencia en la asesoría de OPD?.

Propósitos de la investigación. OPD

1. Comprender qué hace el maestro de

2. Identificar las funciones del asesor de OPD prescritas por el programa educativo

3. Observar cómo resuelve las situaciones a las que se enfrenta enfrenta

4. Tipificar los problemas a los que se

5. Conocer las actividades que mayor incidencia tienen en la formación de las alumnas. 


\section{Justificación}

Desarrollar esta investigación en la escuela normal, específicamente en lo que corresponde al asesoramiento de OPD, tiene gran relevancia, pues destacar las actividades que en lo normativo deben hacerse así como aquellas que por usos y costumbres se ejecutan, darán una visión integral del quehacer del mismo.

Lo anterior brinda en primer lugar en lo personal una idea de la complejidad de la tarea del formador en OPD, en segundo lugar; a los compañeros, pues en el caso de aquellos que no han tenido acceso a este curso es fácil confundir tareas o asignar papeles al asesor de OPD por desconocimiento en la materia. En un tercer momento se beneficia también a las alumnas porque en la medida que el asesor de OPD y el resto de sus asesores conocen esta complejidad ellas recibirán una atención de mayor calidad en todas sus asignaturas.

Con lo anterior no puede sino aspirarse a que el perfil de egreso sea más congruente y consistente en cada una de las alumnas de la escuela normal rural, pues todos sus maestros trabajarán coordinadamente, ya que se pretende que los resultados de esta investigación se den a conocer en la institución donde se labora y se socialice al respecto de los beneficios de conocer o las zonas de riesgo de no conocer el trabajo global que se debe desarrollar con ellas.

La aportación que en lo personal se considera hacer, es la caracterización en esta materia, pues por lo general se ha desarrollado en lo que se percibe guiados en lo normativo y mucho en lo habitual. La sistematización de la presente permitirá recuperar las visiones de quienes viven y desarrollan esta asignatura: docentes y alumnas.

\section{Metodología}

La forma como quedó planteado el problema de investigación en torno a Cuáles son las actividades que el maestro de OPD desarrolla en la escuela Normal Rural Ricardo Flores Magón, es un planteamiento de las ciencias sociales, es un fenómeno social, por lo que el enfoque con el que se abordó es cualitativo.

Se inició examinando el mundo social donde se desenvuelve en su sentido práctico, es decir, donde trabaja; se realizó un proceso inductivo que va de lo particular a lo general. También le caracteriza que no se prueban hipótesis, sino que más bien éstas se generan durante la investigación, autores como Hernández et. al (2008), Martínez (2004) y Álvarez (2006) fundamentaron los planteamientos metodológicos que se siguieron.

Otra de las características de los procesos de indagación seguidos son el conjunto de prácticas interpretativas (en este caso, de asesores de OPD y las propias alumnas de esta asignatura) que hacen visible al mundo, lo transforman y convierten en una serie de representaciones, observaciones, anotaciones, grabaciones y documentos.

El enfoque cualitativo utilizado a veces es referido también como interpretativo o fenomenológico, como su nombre aduce, se enfoca hacia el fenómeno social estudiado y su interpretación. Aballe (2006) hace una descripción de este paradigma y señala que su filosofía es subjetiva, esto porque es desde los sujetos que se trabaja y responde al fenómeno investigado, también advierte lo valioso que en cuanto significado personal adquiere, y con este fundamento, se retoma pues en lo personal se considera que quien mayor conocimiento obtiene de este trabajo es el propio investigador.

El mismo autor menciona que la finalidad de este paradigma es la comprensión, determina significados a las acciones estudiadas y ayuda a explicar el fenómeno que se estudia. Pérez (1990), explica que "no existe una única realidad, sino múltiples realidades interrelacionadas" (p. 27). Como en este caso se explica, es revisar cuál es la percepción que tiene el asesor de 
OPD, pero también sus alumnas acerca de lo que consiste su trabajo y las actividades que desempeña.

Se optó por Método etnográfico porque es el que permite hacer viable la interrogante de investigación de caracterizar la tarea de ser asesor de OPD en la escuela Normal, que es el entorno natural (condición necesaria para este tipo de investigaciones) donde se realiza la práctica docente propia.

La Recolección de datos se desarrolló a través de la encuesta, definida desde la práctica como un cuestionario autoadministrado, autores cono Hernández, et al. (2010) menciona que es un cuestionario que se proporciona directamente a los participantes, quienes a su vez los contestan sin que haya intermediarios, por lo que ellos eligen y escriben sus propias respuestas. Para fines de este trabajo se diseñaron dos tipos de cuestionario: el primero para los profesores asesores de OPD quienes explicitan la experiencia que tienen en esta materia, el segundo para las alumnas quienes a través del mismo señalan y caracterizan a los docentes de OPD que han tenido.

En la recolección de datos se utilizaron las técnicas de observación participante y no participante, se realizaron al interactuar con el grupo investigado (maestros de OPD, en academias) y en su propio ambiente a través de diario de campo recabado con observación no participante, es decir, sin ser parte del grupo.

Autores como Hernández, et al. (2010) explican los papeles del observador donde destaca la no participación como en la contemplación de videos, en este caso; como se explica anteriormente las alumnas realizarán el registro de diario de grupo del cual se recabará información sobre el docente que asesora OPD. En la participante activa o completa es cuando se participa en la mayoría de las actividades y/o se mezcla con el grupo por lo que es un participante más del mismo.

El diario de campo, resultó una herramienta invaluable porque en él se registró todo lo que se observó en el aula. Este instrumento se desarrolló en 3 grupos ajenos y en el propio. En los ajenos, el registro se hizo por parte de alumnas en las que se depositó la confianza para hacerlo por sus habilidades descriptivas. En el grupo propio, se desarrolló por el propio asesor y por una alumna con la finalidad de tener elementos de triangular la información.

En cuanto a la unidad de análisis, se consideró que la integrara el propio investigador el resto de docentes que tiene a su cargo la asignatura de OPD en relación con los grupos que se atienden en la escuela normal en este programa educativo, como se indica en la tabla 3.

Tabla 3. Unidad de análisis.

\begin{tabular}{ccc}
\hline Docente & Grupo & No. Alumnas \\
\hline $\mathbf{1}$ & 1 A Preescolar & 32 \\
$\mathbf{2}$ & 2 A Preescolar & 25 \\
$\mathbf{3}$ & 3 A Preescolar & 33 \\
$\mathbf{4}$ & 3 B Preescolar & 31 \\
\hline
\end{tabular}


Como se observa, son un total de 121 alumnas que auxilian a caracterizar las actividades que el asesor de OPD desarrolla, así como los 4 asesores darán su información a través del cuestionario diseñado para tal fin.

Otro aspecto metodológico es el método por el cual se realizó el análisis de la información recabada, que en este caso fue a través del método de sistematización de la práctica docente de Mercedes Gagneten (1994), quien establece siete fases para ello.

La primera es la reconstrucción de la práctica, hecha con técnicas e instrumentos mencionados, enseguida se realiza un análisis de la información en la que se tematiza con la información recurrente y se forman categorías o temas abarcativos. La tercera fase se denomina interpretación, y se define como una síntesis personal acerca de lo que se observa en la red o tema encontrado y se confronta con marcos teóricos consultados o se busca nueva teoría para respaldar dicha interpretación, cuidando que no quede en el sentido común.

En un cuarto momento, se exige al investigador realizar otro ejercicio de síntesis personal y elaborar un constructo de lo que su categoría trató en el aquí y ahora, a este momento se le denomina conceptualización. La quinta fase es la generalización, está determinada como el deber ser construido, es decir lo que debe ser en cualquier espacio, por lo que se considera es el deber ser.

La sexta fase son las conclusiones, aquí se dan respuesta a las preguntas de investigación y consideraciones acerca del proceso de investigación. Mientras que la séptima fase es la propuesta, de toda investigación se derivan elementos propositivos que el autor debe abstraer y hacerlos explícitos tanto para sí mismo como para sus participantes en la investigación o para cualquier lector que se acerque a su investigación.

\section{Resultados preliminares}

Los hallazgos que se presentan en la figura $1 \mathrm{y}$ 2 exponen la percepción que se tiene de dos ámbitos que se identifican a cerca de las funciones del asesor de los cursos de acercamiento a la práctica docente en la escuela normal. El primero de ellos es el que se relaciona con las actividades o funciones eminentemente académicas y normativas que realiza el asesor para el desarrollo de dichos cursos. El segundo presenta una serie de funciones que son de carácter extracurricular no académicas en las que el docente, por las obligaciones contraídas dentro de la escuela debe desarrollar para complementar la formación profesional de las estudiantes.

Los elementos principales de la caracterización de la función de asesoría, que fueron identificados por el proceso de esta investigación son los siguientes:

- Espacio para articular los conocimientos en diferentes materias

- Planificación, observación, diálogo con los niños y niñas

- Análisis, reflexión e interpretación de la experiencia

- Poner en práctica conocimientos del semestre

- Pautas para organizar jornadas de observación y práctica

- Desarrollo de competencias didácticas así como de la sensibilidad necesaria para trabajar con los niños, así como establecer relaciones de colaboración con otros profesores, padres y alumnos

- Focalización hacia las actividades que realizan los niños durante su estancia en los planteles. 
RECIE. Revista Electrónica Científica de Investigación Educativa Vol. 1, núm. 2, enero-diciembre 2013, pp. 331-337.

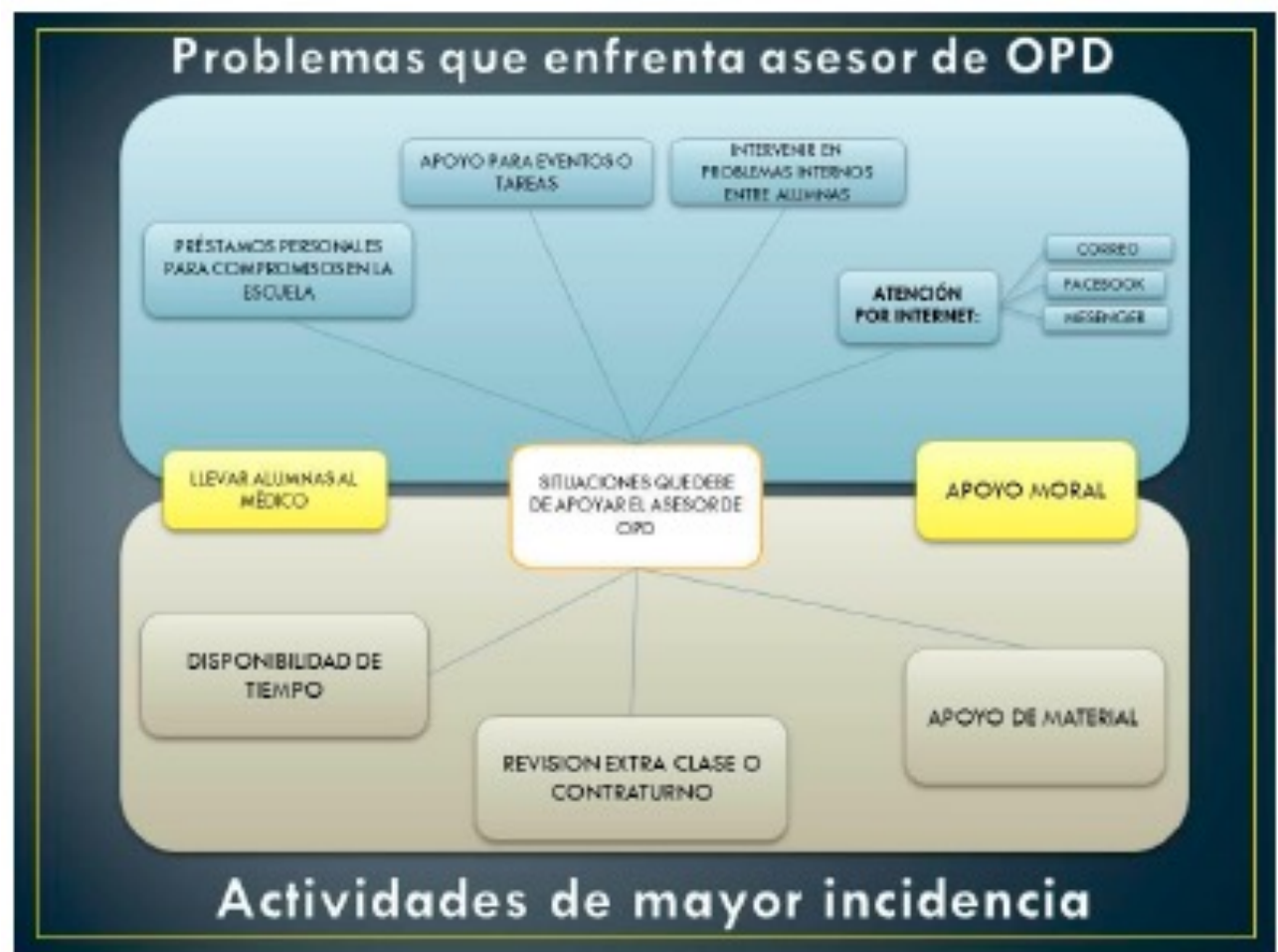

Figura 1. Problemas y actividades del asesor.

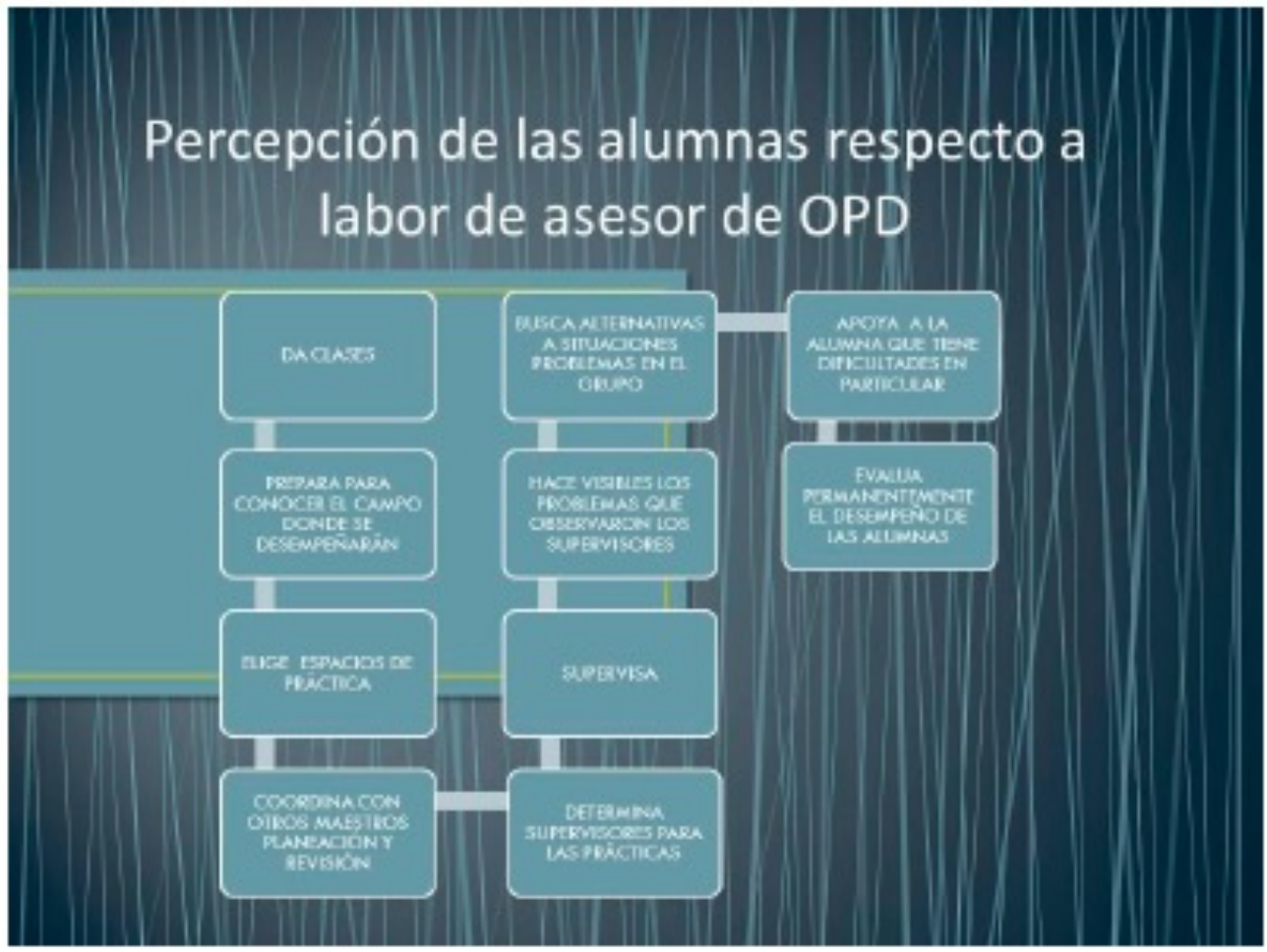

Figura 2. Percepción de las alumnas 


\section{Conclusiones}

- El asesor es observado desde diferentes perspectivas por las alumnas, de pronto sólo es el asesor del aula cuya incumbencia llega hasta ahí

- Por otra parte, el asesor se convierte en tutor de cada una de sus alumnas y les va guiando positivamente en el camino de formación docente

- El asesor es también un tutor que auxilia en cada una de las contingencias cotidianas que la alumna vive en el internado, cuya naturaleza le da una complejidad específica

- Son múltiples las tareas que el asesor de OPD realiza con su grupo tutorial

\section{Referencias}

Aballe, V. (2007). Metodología de la investigación educativa. Habana, Cuba. Universidad de la Habana. fedvirtual2.fed.uh.cu/dspace/bitstrea $\mathrm{m} /$.../1/Tesis+maestríaAlea.doc

Alvarez, G. J, J. L.(2006). Cómo hacer investigación cualitativa. Fundamentos y metodología. México. Editorial Paidós.

ANUIES. (2000). Programas institucionales de tutoría. Una propuesta de la ANUIES para su organización y funcionamiento en las instituciones de educación superior, México. ANUIES.

Gagneten, M. M. (1990). Hacia una metodología de sistematización de la práctica. Buenos Aires Argentina. Hvmanitas
García, B. F.J. y Fortea B. M.A. (2006). Contrato pedagógico. España. Universidad Jaume

Goetz J.P. y Lecompté M.D. (1988). Etnografía y diseño cualitativo en investigación educativa. Madrid. España. Ediciones Morata.

Hernández, S. R. Fernández, C. C. Baptista, L.P. (2005) Metodología de la investigación. México. Editorial Mc. Graw Hill.

Lella, C. (1999). Modelos y tendencias de la formación docente. Lima Perú. OEI

Martínez M, M.(1998) La investigación cualitativa etnográfica en educación. Manual teórico práctico. Tercera edición. Editorial trillas.

Papalia, D. E. S. W. O, R. D. F. (2010). Desarrollo humano. Editorial Mc. Graw Hill. Undecima edición. México.

Pérez, G. A. (1999). La cultura escolar en la sociedad neoliberal. España. Editorial Morata.

Porlán, R. M. J. (2000). El diario del profesor: un recurso para la investigación en el aula. Sevilla: Diada. FernándezBallesteros. http://www.librospdf.net/rafaelporlan-diario-de-un-profesor/1/

Rodríguez, G. G. J, G. F. y E,G. J.. (1999). Metodología de la investigación cualitativa. Málaga. España. 1999. Segunda edición. Ediciones Aljibe.

SEP. (2004). Panes y programas de estudio. México

Woods, P. (2006). Reseña de la escuela por dentro. La etnografía en la investigación educativa. Mérida Venezuela. Educere. Universidad de los Andes. 
RECIE. Revista Electrónica Científica de Investigación Educativa Vol. 1, núm. 2, enero-diciembre 2013, pp. 331-337. 